\title{
Gruss vom Planeten NHS
}

\section{Xaver Huber}

Dr. med., Facharzt für Chirurgie

Die angelsächsischen Spitäler sind uns zeitlich voraus. Manches aus dem Erfahrungsbericht kennt man aus Schweiz, anderes ist (noch) unvorstellbar. Ärzte müssen bestimmte finanzielle Ziele erfüllen, zahlreiche Instanzen überprüfen die Qualität, die Bereiche sind stark segmentiert, hohe Fallzahlen ermöglichen hohe Expertise. Ständig wird hinterfragt: Ist eine Behandlung objektiv wirksam?

Ich arbeitete während eines Jahres am Frimley Park Hospital in Frimley, Grafschaft Surrey, als Specialty Registrar auf der Allgemeinchirurgie. Frimley Park Hospital ist ein NHS Trust Hospital mit rund 750 Betten und entspricht dem Leistungsspektrum eines grossen Schweizer Kantonsspitals mit einem Einzugsgebiet von rund 400000 bis 500000 Einwohnern und rund 4500 Angestellten. Das Spital wickelt etwa 100000 Notfallkonsultationen ab und verzeichnet etwa 30000 Notfalleintritte jährlich. Es bietet u.a. 24hNotfallversorgung für Gefässchirurgie, Stroke, Kardiologie sowie Allgemeinchirurgie. Es hat hingegen keine Neuro-, Thorax- und Herzchirurgie [1]*.

\section{NHS - ubiquitär und kolossal}

Der National Health Service (England, respektive ihre Pendants Scotland, Wales und Ireland) ist eine staatliche Organisation, welche die Gesundheitsversorgung Englands organisieren und sicherstellen muss, und deren Dienstleistungen allen in England wohnhaften Menschen kostenlos zur Verfügung stehen. Der NHS wird direkt aus Steuergeldern finanziert, machte im Jahr 2012/2013 rund 108 Milliarden Pfund (ungefähr 160 Milliarden Franken!) Umsatz und deckt die Versorgung von rund 63 Millionen Menschen ab [2].

Die Versorgung wird neu organisiert durch territorial definierte Clinical Commissioning Groups (CCG) [3],
Die Literatur findet sich unter www.saez.ch $\rightarrow$ Aktuelle Ausgabe oder $\rightarrow$ Archiv $\rightarrow 2015 \rightarrow 47$.

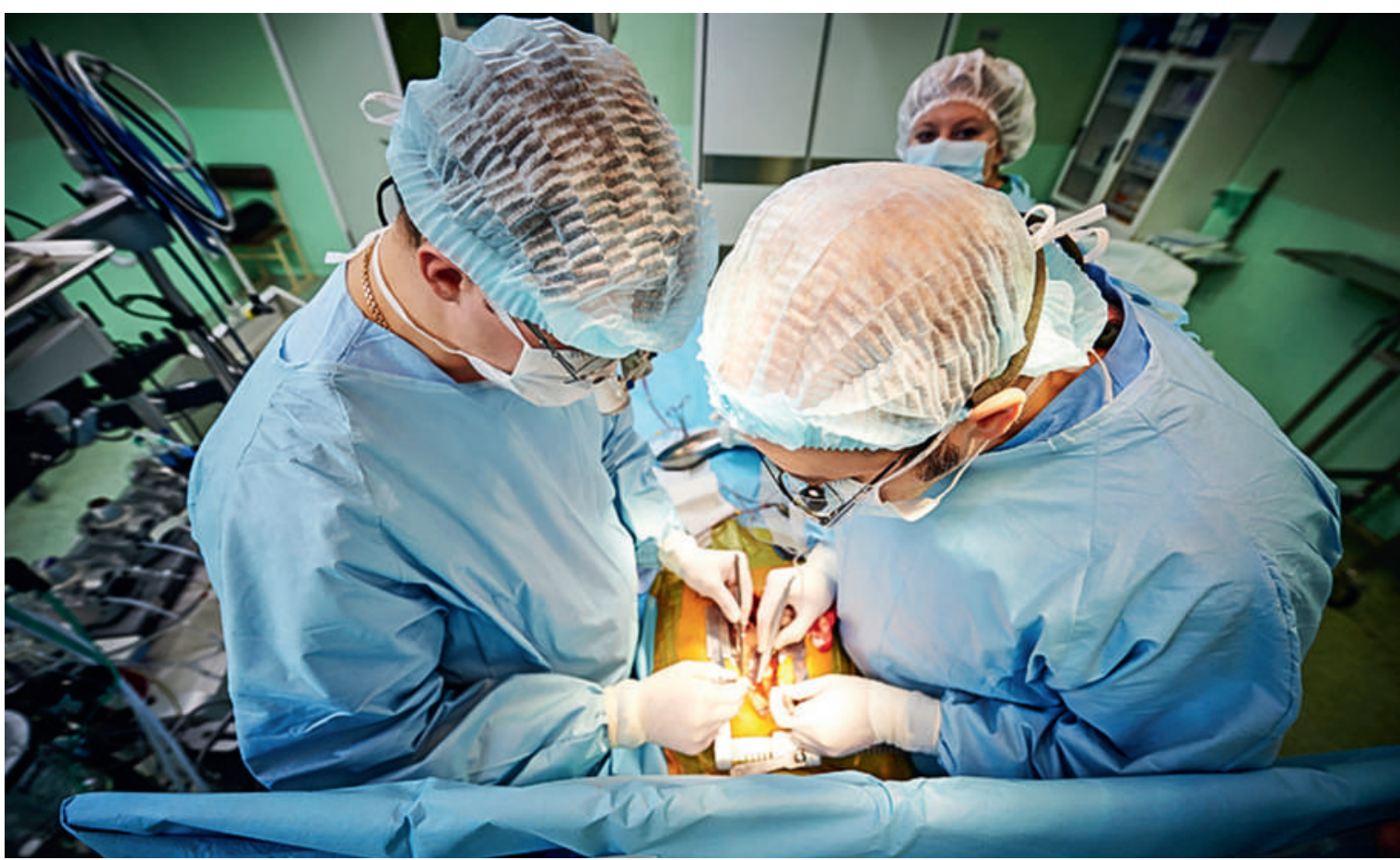

Das Prinzip heisst Fliessband: Damit der Einzelne möglichst viel vom Gleichen bewältigen kann, wird die Arbeit durch Spezialisierung segmentiert. 
die Leistungserbringer bezahlen, d.h. die Leistung einkaufen bei den Spitälern, die meist in sogenannten NHS Trusts organisiert sind. Die CCGs bestehen aus allen GPs (general practitioners) und weiteren Healthcare Professionals des spezifischen Gebietes. Die GPs haben über die CCGs Einfluss auf die Mittelallokation. Zugleich ist der einzelne Patient an einen bestimmten general practitioner gebunden, bei dem er sich registrieren lassen muss, um über dessen Zuweisung zum entsprechenden Spezialisten Zugang zu erlangen. Damit ist England im Prinzip eine einzige riesige HMOPraxis. Der ambulante Sektor ist vollständig von den GPs dominiert, freipraktizierende Gynäkologen und Gynäkologinnen beispielsweise gibt es kaum. Es existiert ein privater Gesundheitssektor, der aber weder anteilsmässig noch qualitativ (i.e. Grösse der Risiken und Komplexität) mit dem schweizerischen vergleichbar ist.

\section{England ist im Prinzip eine einzige riesige HMO-Praxis.}

Im Land der Business Schools ist die Gesundheit organisiert wie ein beliebiger Industrieprozess. Standards und Guidelines und deren Durchsetzung geniessen einen sehr hohen Stellenwert. Jeder Trust und jeder GP hat bestimmte finanzielle Ziele zu erfüllen, und der finanzielle Druck ist erheblich. So hat die letzte Reform der Regierung Cameron das Budget des NHS um rund 20 Milliarden Pfund in vier Jahren gekürzt [4]. Es ist das erklärte Ziel aller politischen Kräfte, und dies entspricht der effektiven Erwartungshaltung in der Gesellschaft Grossbritanniens, dass alle den gleich guten Zugang zu Gesundheitsdienstleistungen haben und alle gleich behandelt werden. Dies vor dem Hintergrund permanenter und scharfer öffentlicher Kritik am NHS, er sei ineffizient, langsam und bürokratisch. Das System steht dermassen unter Dauerkritik, dass ein Paralleluniversum aus Instanzen, die die Leistung der Trusts und CCGs kontrollieren, entstanden ist. Um einige zu nennen: GMC (General Medical Council registriert Ärztinnen und Ärzte und kontrolliert deren Qualifikation), MONITOR (Kontrollorgan des Gesundheitsministeriums) oder das NICE (National Institute for Health and Care Excellence), das Behandlungsstandards definiert. Die Care Quality Commission [5] (CQC) führt unangemeldete mehrtägige Spital-, aber auch GPInspektionen (i.e. Hausarztinspektionen) durch und verfasst umfassende Berichte. Ich lade die Leserschaft ein, die entsprechenden Berichte auf www.cqc.org.uk/ einzusehen.

Ein weiteres Mittel, die Qualität des Systems zu überprüfen, sind sogenannte Audits, die auf allen Ebenen und hinsichtlich aller Fragestellungen permanent durchgeführt werden. Hinzu kommen schliesslich zahlreiche (private und staatliche) Initiativen, Kampagnen und Institutionen, die unabhängig von den grossen offiziellen Qualitätssicherungsinstrumenten permanent das Licht der Öffentlichkeit suchen. Somit kann der NHS durchaus als eine Art Modell betrachtet werden. Auf ihm lastet der Anspruch, die beste Medizin zu leisten unter maximalem ökonomischem Druck.

\section{Und - on the ground?}

An einer Einführungsveranstaltung wurde vom SpitalCEO deklariert: "We are a management-led hospital.» Und dies ist keine Worthülse, sondern beschreibt die Situation, in der sich die Ärztinnen und Ärzte befinden. Sie werden verstanden als Angestellte eines Unternehmens, die in der Chirurgie beispielsweise klare Umsatzziele erreichen müssen, was durchaus Sinn macht, da die knappen Ressourcen auf maximal viele Patienten angewendet werden sollen (Versorgungssicherheit). Wenn ein Saal sechs Cholezystektomien pro Tag hergibt, dann muss diese Kapazität auch ausgelastet werden, um eine lange Warteliste abzuarbeiten. Was passiert aber, bezogen auf die Schweiz, wenn ein Umsatzziel gesetzt wird in einem Umfeld scheinbar unlimitierter Ressourcen?

\section{Die Care Quality Commission führt unan-} gemeldete mehrtägige Spital-, aber auch Hausarztinspektionen durch.

Der NHS ist Meister im Definieren von Richtlinien, wie eine Gesundheitsdienstleistung zu erfolgen hat. Diese Richtlinien und Pathways werden für jedes Problem fortlaufend entwickelt, evaluiert und verfeinert. Auf der expliziten Ebene sollen sie helfen «to keep patients safe». Immer wird gefragt: Ist eine Prozedur, eine Behandlung oder eine Pflege safe? Es wird auch ständig hinterfragt: Ist eine Behandlung objektiv wirksam? Implizit aber schwingt mit: Wie weit kann man gehen oder: Wie billig kann man's haben? Nehmen Sie laparoskopische Cholezystektomien. Es gibt umfassende Literatur, dass man eine Gallenblase als tageschirurgischen Fall operieren und den Patienten bzw. die Patientin am gleichen Tag entlassen kann. Entsprechend wird die Gallenblasenentfernung als tageschirurgischer Fall geplant und durchgeführt. Das bedeutet realiter einen Spitalaufenthalt von rund 6-8 Stunden. Die Rate an Patienten, die bleiben müssen, die also einen "failed discharge» repräsentieren, beträgt circa 20\% [6]. Das deckt sich mit meinen persönlichen Erfahrungen. Man stelle sich eine Patientin in der Schweiz vor, die vier 


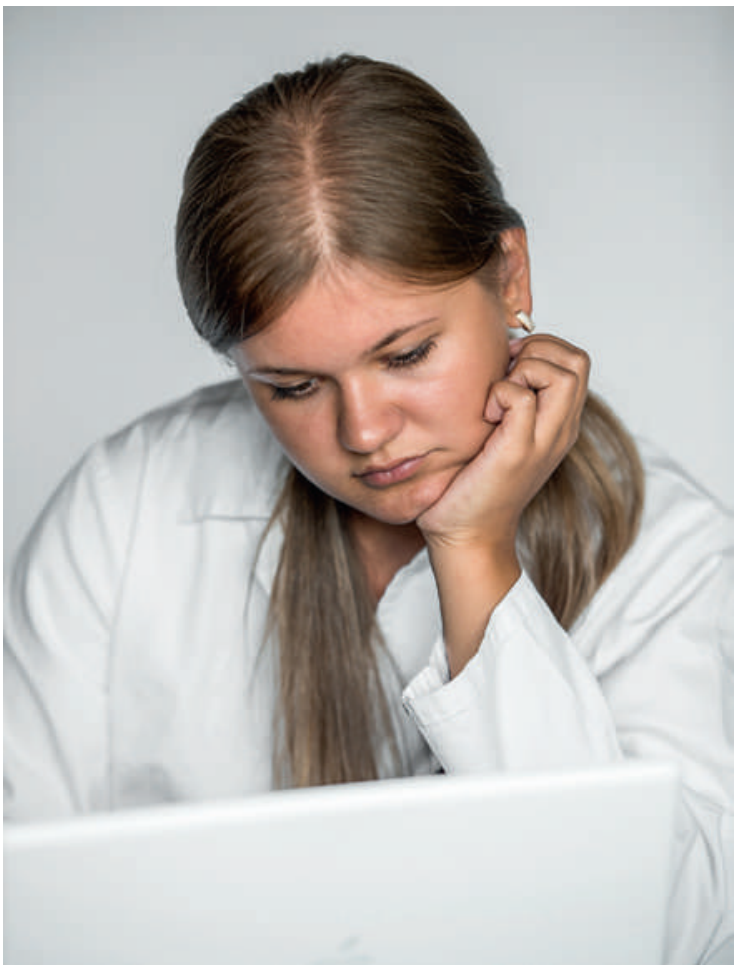

Junge Ärztinnen und Ärzte werden mit hochgradig prozeduralisierten Vorgängen wie Briefe-Schreiben oder Daten-Eingeben beschäftigt - nicht besonders motivierend. Erfahrene Ärzte sollen die Behandlungsqualität sichern.

Stunden nach Erwachen aus der Narkose nach einer Gallenblasenoperation auf der Strasse steht. Die Frage ist nicht, ob es geht. Ich kann Ihnen versichern, es geht. Die Frage ist: Wie lange können - wollen - wir uns die Übernachtung noch leisten?

\section{Spezialisierung, Prozeduralisierung und Seniorisierung}

Das Prinzip heisst Fliessband, und damit ein einzelner Angestellter (ob Arzt, Krankenschwester oder Pflegehilfe) möglichst viel vom Gleichen bewältigen kann, wird die Arbeit durch Spezialisierung segmentiert. Der phlebotomist beispielsweise macht nichts anderes, als morgens auf der Abteilung Blut abzunehmen. Die Pflegenden sind im NHS straff hierarchisch organisiert und sind im Spital an einer strikten Uniformierung in verschiedenen Grau- und Blautönen zu erkennen. Einzelnen specialist nurses obliegt es sogar, eigene Sprechstunden abzuhalten. Sie betreuen meist eine spezifische Gruppe von Patienten analog zu den entsprechenden Fachärzten (und jeweils diesen zugeordnet und rechenschaftspflichtig), führen Endoskopien oder auch Sonographien durch. Spezialisiert sind natürlich auch die Ärztinnen und Ärzte, in der Chirurgie zum Beispiel ist die Aufteilung in upper and lower gastrointestinal surgery etabliert (um nur einmal die plumpste Segmentierung zu erwähnen). Damit können Chirurgen in einem spezifischen Bereich grosse Fallzahlen aufweisen, und entsprechend hoch und differenziert ist ihre prozedurale Expertise. Doch der Patient bezahlt einen Preis für die Hochspezialisierung. Die einzelnen Spezialisten büssen sukzessive die Fähigkeit ein, im Dienst ein breites Spektrum von Problemen abzudecken [7, 8]. Die prozedurale Spezialisierung führt auch zur intellektuellen Abschottung gegen alles, was nicht genau in den eigenen Fachgarten passt. Englische general surgeons schauen Röntgenbilder von Knochen nicht einmal von weitem an. Ärztinnen und Ärzte, die in Spitälern arbeiten, sind also sowohl der Prozeduralisierung als auch der Segmentierung unterworfen, in England noch viel mehr als in der Schweiz. Etwas Drittes folgt aus dem zunehmenden Druck auf die Spitäler, bessere Qualität zu liefern und wirtschaftlicher zu arbeiten: die Seniorisierung. Im besagten Spital darf ein Assistenzarzt im ersten Jahr keine Visite alleine machen, sondern es wird gefordert, dass er von einem Registrar oder einem mindestens mehrjährig Erfahrenen begleitet wird, der ihm dann sagt, was er zu tun hat. Daher: Der Assistent spricht kaum mehr selber mit dem Patienten, sondern rennt herum wegen Blutabnahmeformularen, INR-Einstellungen und verfasst Austrittsbriefe. Auf der Notfallstation in Frimley war eben eingeführt worden, dass rund um die Uhr ein $A \& E$ consultant (in etwa ein Leitender Arzt der Notfallmedizin) auf der Notfallstation anwesend sein muss. Das Management weiss, dass Entscheide schneller zustande kommen, je ranghöher der Arzt oder die Ärztin ist, der oder die auch initial schon am Bett steht. Mehr Effizienz im Ablauf wird dadurch erreicht, dass man sich möglichst viele nicht abschliessende Entscheidungsträger ausspart - d.h. die jüngeren Ärzte und Ärztinnen. Diese beschäftigt man mit hochgradig prozeduralisierten Vorgängen wie

\section{Die einzelnen Spezialisten büssen sukzessive} die Fähigkeit ein, im Dienst ein breites Spektrum von Problemen abzudecken.

Briefe schreiben, Computer mit Daten füttern, bereits definierte Medikationsschemata (Insulin, Marcoumar usw.) für die Pflege unterschreiben. Die Seniorisierung verbessert kurzfristig zwar - vielleicht - die Qualität und die Effizienz der Dienstleistung, sie führt aber bei den jüngeren Ärztinnen und Ärzten zu einer massiven Abnahme der Identifikation mit ihrer Arbeit. 


\section{Effekte auf die Risikoselektion - oder: Wer ist schuld?}

Im NHS wird sehr risikobewusst operiert. Bei Patienten mit nur den geringsten Hinweisen auf eine prognoserelevante Komorbidität wird ein Eingriff als problematisch angesehen. So kann es passieren, dass eine rund 80-jährige Patientin wegen rezidivierender Cholezystitiden im gleichen Halbjahr zwei Cholezystostomien erhält, anstatt operiert zu werden. Dies passiert in einem System, in dem Mortalitätsraten als Gradmesser chirurgischer Qualität genommen werden. Die Mortalitätsraten jedes einzelnen Consultants der Chirurgie werden durch die Fachgesellschaften online ausgewiesen und sind für jeden einsehbar. So entsteht ein erheblicher Druck auf den einzelnen Chirurgen, Risiken zu vermeiden, selbst dann, wenn der Patient den Eingriff eigentlich braucht. Das geben die englischen Chirurgen im Vieraugengespräch auch zu. Denn: Stirbt ein 80-Jähriger an einer perforierten Sigmadivertikulitis ohne Operation, ist die Krankheit schuld, stirbt er nach der Operation, ist es die Schuld des Chirurgen.

Ich bin auch auf Patienten gestossen, deren Kolon- und Rektumkarzinome lediglich mit Stomata versorgt worden waren. Anfänglich glaubte ich, es handle sich um ein lokales Phänomen. Jedoch: Gemäss des National Bowel Cancer Audit 2013 wurden in England 38\% (!) aller bewiesenen Kolonkarzinome nicht operiert. Für NO- und MO-Tumoren wird immer noch eine NichtOP-Rate von 25\% ausgewiesen [9]. Der Bericht liefert für diese Rate keine plausible Erklärung. Zum Vergleich: Eine deutsche Studie weist eine Operationsrate von kolorektalen Karzinomen in Deutschland von über $90 \%$ aus [10]. So verwundern einen Schlagzeilen wie Doctors are blamed for elderly cancer deaths [11] überhaupt nicht mehr. Bevor die geneigte und fachkundige Leserschaft in Empörung ausbricht: Haben Sie sich schon einmal gefragt, wie hoch die 30-Tages-Mortalität eines 80-Jährigen ist nach einer Kolonresektion [12]?

\section{Medical Emergency Teams - zwischen REA und Ruhe: Hintergründe verstehen}

Englische Spitäler sind gut gefüllt mit Patienten, und gelegentlich auch richtiggehend überrannt. Dem gegenüber steht jeweils ein im Verhältnis zur Schweiz kleines Grüppchen von Ärztinnen und Ärzten. Die Patienten liegen dicht gepackt in 6er-Kojen, es ist eng und verhältnismässig laut, und nicht alle Patienten hatten im besagten Spital Tageslicht. Patienten können da schon mal vergessen gehen oder werden nicht adäquat beurteilt aus Mangel an Zeit, Raum und Ruhe. Eine typische NHS-Antwort auf ein solches Problem ist die Etablierung sogenannter
MET (medical emergency teams) oder RRT (rapid response teams). Diese Teams bestehen aus ärztlichen und pflegerischen Vertretern verschiedener Fachrichtungen, typischerweise von der Anästhesie, Intensivmedizin, Inneren Medizin und manchmal auch Chirurgie. Die Pflege überwacht Patienten anhand vorgegebener Vitalparameter wie Sättigung, Atemfrequenz, Blutdruck, Herzfrequenz und GCS und erfasst die Resultate in einem Scoring-System. Erreicht ein Patient einen Schwellenwert, wird der MET call automatisch ausgelöst und zwingt die genannten Fachrichtungen ans Bett des Patienten, im Grunde verhält es sich damit wie mit einem REA-Alarm light. Typischerweise ist dann eben niemand des betreuenden (ärztlichen) Teams bei einem solchen MET-Call dabei, und das zuständige Team muss sich anhand der Akten ein Bild machen und Massnahmen treffen, um den Zustand des Patienten zu verbessern. Es wird postuliert, dass solche MET calls unnötige ITU admissions, Herzstillstände und die Zahl postoperativer Komplikationen verhindern, was sicherlich für ein System, das personell notorisch unterdotiert ist, Sinn ergibt. Die METs sind von den einzelnen Fachrichtungen unabhängig, und es kann schon irritierend sein, ein fremdes Behandlungsteam am eigenen Patienten anzutreffen, für dessen Problem man vielleicht eine Erklärung hat und deshalb sich vom Abweichen des mechanistisch angewandten Scores nicht beein drucken lässt. Auch fliessen Schmerzen nicht in diesen Score ein. Einen Herzinfarkt verpasst man spielend.

\section{Es entsteht erheblicher Druck für den} Chirurgen, Risiken zu vermeiden, auch wenn der Patient den Eingriff eigentlich braucht.

Wer sich für sein Spital Gedanken macht, ob er ein medical emergency team braucht, muss sich fragen, ob das zuständige Team, und das betrifft sowohl die ärztliche als auch die pflegerische Seite, im Verhältnis zum Schweregrad der Erkrankungen personell hoch genug dotiert ist und ob die Arbeitsprozesse wirklich patientenzentriert sind (Stichwort: Pflege am Computer). Die Einführung von METs ist meines Erachtens eine taugliche, aber defensive Massnahme, um einen Verlust an Patientensicherheit zu verhindern in einer Situation zunehmender Ressourcenknappheit (Raum und Zeit pro Patient). Ihre Einführung ist in meinen Augen kein Qualitätsmerkmal.

Der angelsächsische Raum ist uns zeitlich voraus. Manches wird die Leserschaft wiedererkannt haben, anderes ist noch unvorstellbar. Wir können von anderen lernen, doch wir sollten uns vor blinder Übernahme fremder Konzepte hüten.

\section{Bildnachweis}

(c) Dmitry Kalinovsky | Dreamstime.com (Operation)

(C) Victorpr | Dreamstime.com (Ärztin) 
1 www.frimleypark.nhs.uk

2 www.nhs.uk/NHSEngland/thenhs/about/Pages/overview.aspx

www.nhs.uk/Service-Search/Clinical-Commissioning-Group/

4 www.theguardian.com/society/2011/oct/17/nhs-cuts-impact-onpatients-revealed

5 www.cqc.org.uk

6 Cochrane Database Syst Rev. 2008 Jul 16;(3):CD006798-

7 www.rcseng.ac.uk/policy/documents/RCS\%2OEmergency\%20 surgery\%20policy\%20briefing.pdf
8 Concerns about UK emergency surgery death rates. Lancet. 2014;384(9950):1238

9 www.hscic.gov.uk/bowel

10 Kleespies A, von Dossow-Hanfstingl V, Renz B, Khalil P, Angele MK Schon zu alt für eine OP? Chirurg, 2013;84:296-304.

11 The Times, 24.1.2014.

12 Kiran RP, Attaluri V, Hammel J, Church J.A novel nomogram accurately quantifies the risk of mortality in elderly patients undergoing colorectal surgery. Ann Surg. 2013;257:905-8. 